[0212-7199 (2007) 24: 6; pp 263-266] ANALES DE MEDICINA INTERNA Copyright (C) 2007 ARAN EDICIONES, S.L.

An. MED. InTERNA (Madrid) Vol. 24, N. ${ }^{\circ} 6$, pp. 263-266, 2007

\title{
Factores de riesgo y pie diabético
}

\author{
V. MARTÍN BORGE, L. HERRANZ DE LA MORENA, I. CASTRO DUFOURNY, \\ A. FERNÁNDEZ MARTÍNEZ, L. F. PALLARDO SÁNCHEZ
}

\author{
Unidad de Diabetes. Hospital Universitario La Paz. Madrid
}

DIABETIC FOOT AND RISK FACTORS

\section{RESUMEN}

Objetivo: Identificar factores clínico-metabólicos relacionados con la neuropatía diabética y la enfermedad arterial periférica en pacientes evaluados en una unidad de pie diabético.

Método: Desde 2000 a 2005 se evaluó, en una población de 304 diabéticos, la presencia de neuropatía (monofilamento, diapasón graduado y escala clínica de Boulton) y de enfermedad arterial periférica (índice tobillo-brazo y/o dedo-brazo). Se clasificaron los pacientes en cuatro grupos: sin patología (grupo normal), con neuropatía (grupo neuropático), con enfermedad arterial periférica (grupo vascular) y con ambas patologías (grupo mixto). Se compararon las características de los pacientes de cada grupo. Se recogieron las variables: edad, sexo, tipo de diabetes, duración, complicaciones microvasculares y macrovasculares, hipertensión, dislipemia, tabaquismo, antiagregación y HbA1C media del último año.

Resultados: Comparado con el grupo normal, la edad media, la frecuencia de hipertensión y la de cardiopatía isquémica fue significativamente mayor $(\mathrm{p}<0,005)$ en el grupo vascular y mixto $(55 \pm 14$ vs. $63 \pm$ 13 y $65 \pm 10 ; 45,5 v s .69,2$ y $70,3 \% ; 23,8 v s$. 46,2 y $39,2 \%$, respectivamente). Comparado con el grupo normal, la frecuencia de retinopatía , la de nefropatía y HbA1c media fue significativamente mayor $(\mathrm{p}<0,05)$ en el grupo neuropático y mixto $(32,7 v s .62,5$ y $66,2 \% ; 24,8 v s .45,3$ y $47,3 \% ; 7,4 \pm 1,2$ vs. $8,1 \pm 1,6$ y $8,0 \pm 1,3$ respectivamente).

Conclusiones: Este estudio indica que el desarrollo de neuropatía diabética está en relación con un peor control metabólico y con la existencia de otras complicaciones microvasculares; mientras que la edad, la hipertensión y la enfermedad coronaria constituyen factores de riesgo para la coexistencia de arteriopatía periférica.

PALABRAS CLAVE: Diabetes Mellitus. Neuropatía periférica. Arteriopatía periférica. Factores de riesgo.

\section{ABSTRACT}

Objective: To identify clinic and metabolic risk factors for diabetic neuropathy and peripheral arterial disease in patients evaluated in a diabetic foot unit care.

Method: From 2000 to 2005 we evaluated the presence of diabetic neuropathy (monofilament, tuning fork and Boulton's clinic scale) and peripheral arterial disease (ankle-brachial index and toe-brachial index) in 304 diabetic patients. We classified patients in four groups: patients without pathology (normal group), with neuropathy (neuropathic group), with peripheral arterial disease (vascular group) and with both pathologies (mixed group) and we compared the characteristics of each group. We analysed other poblational characteristics: age, gender, type of diabetes, duration, microvascular and macrovascular complications, hypertension, smoking habit, antiagregation and mean HbAlc in the last year.

Results: Age, frequency of hypertension and coronary disease were significantly higher $(p<0.005)$ in vascular and mixed group than in normal group $(63 \pm 13$ and $65 \pm 10 \mathrm{vs} .55 \pm 14 ; 69.2$ and $70.3 \mathrm{vs} .45 .5 \%$; $46,2 \%$ and $39.2 \%$ vs $23.8 \%$, respectively). Frequency of retinopathy, nephropathy and HbAlc were significantly higher $(p<0.05)$ in neuro-

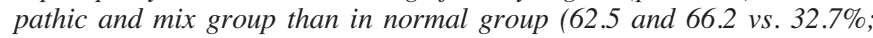
45.3 and 47.3 vs. $24.8 \% ; 8.1 \pm 1.6$ and $8.0 \pm 1.3$ vs $7.4 \pm 1.2$ respectively).

Conclusion: This study indicates that the development of diabetic neuropathy is related with worse metabolic control and the presence of other microvascular complications; while age, hypertension and coronary disease are risk factors for peripheral arterial disease.

KEY WORDS: Diabetes mellitus. Peripheral neuropathy. Peripheral arterial disease. Risk factors.

Martín Borge V, Herranz de la Morena L, Castro Dufourny I, Fernández Martínez A, Pallardo Sánchez LF. Factores de riesgo y pie diabético. An Med Interna (Madrid) 2007; 24: 263-266.

\section{INTRODUCCIÓN}

El pie diabético es una complicación crónica de la diabetes de gran importancia desde un punto de vista médico y social, ya que en nuestro medio, constituye la causa no traumática más frecuente de amputación de miembros inferiores (1).

La existencia de neuropatía periférica, de enfermedad arterial periférica y de alteraciones biomecánicas hacen que el pie de los pacientes diabéticos sea menos sensible, esté peor vascularizado y presente zonas de descarga anómalas, siendo más

Trabajo aceptado: 5 de febrero de 2007 
susceptible de padecer lesiones ulcerativas graves con gran tendencia a la infección.

Existe evidencia clínica de que la aparición de las complicaciones microvasculares de la diabetes como la retinopatía, la nefropatía o la neuropatía está en relación con el control metabólico (2-4). Algunos estudios han ido más allá estableciendo una relación entre la severidad de la neuropatía y la duración del mal control metabólico (5).

Por otro lado se ha demostrado que el aumento de la incidencia de arteriosclerosis de los pacientes diabéticos se relaciona entre otros factores con la dislipemia, la hipertensión y la hiperglucemia crónica, en definitiva, con el mal control metabólico de la diabetes $(6,7)$.

El objetivo de este estudio ha sido identificar las condiciones clínicas y epidemiológicas que hacen más vulnerables a los pacientes diabéticos para sufrir complicaciones vasculares y neuropáticas que conducen al desarrollo del pie diabético.

\section{MÉTODO Y PACIENTES}

Desde enero de 2000 hasta diciembre de 2005 se evaluaron ,en una Unidad de pie diabético, 304 pacientes diabéticos (606 miembros ya que dos de nuestros pacientes habían sufrido previamente una amputación) con las siguientes características: edad media de 59,9 \pm 13 años, 174 varones y 130 mujeres, 92 con diabetes tipo 1 y 212 con diabetes tipo 2 y con una duración media de la diabetes de 16,2 \pm 11,6 años. Estos pacientes fueron seleccionados para realizar el estudio teniendo en cuenta la presencia de al menos uno de los factores predisponentes para la aparición de pie diabético: edad, tiempo de evolución de la diabetes superior a 10 años, presencia de otras complicaciones micro y macrovasculares, mal control metabólico, alteraciones biomecánicas y úlceras previas.

Se valoró la presencia de neuropatía mediante tres métodos: escala clínica de Boulton (8), sensibilidad vibratoria con el diapasón graduado de $128 \mathrm{Mhz}$ en el dorso del primer dedo de cada pie y sensibilidad epicrítica con el monofilamento de 10 gramos en 5 puntos de la planta de cada pie.

Se consideró pie neuropático aquel que presentó alguna de las tres pruebas patológicas: 5 o más puntos en la escala de Boulton, sensibilidad vibratoria inferior a 4 o percepción de menos de 4 de los 5 puntos explorados con el monofilamento.

Para la valoración de la existencia de enfermedad arterial periférica se determinaron los índices tobillo-brazo (ITB) y dedo-brazo (IDB) utilizando para ello un doppler con una frecuencia de emisión de $8 \mathrm{Mhz}$ y un manguito para la toma manual de presión arterial (de $10 \mathrm{~cm}$ para el ITB y de $25 \mathrm{~mm}$ para el IDB). La determinación de la presión arterial se realizó a nivel de la arteria braquial en ambos brazos, a nivel de la arteria tibial posterior en ambos tobillos, de la arteria tibial anterior en el dorso de cada pie y a nivel de la arteria pedia dorsal en el dorso del primer dedo de cada pie (9).

Para el cálculo del ITB se utilizó la presión arterial braquial más elevada o la más cercana a la toma maleolar. De los cuatro valores obtenidos de ITB (toma braquial con las tomas de las dos arterias tibiales posteriores y con las de las dos arterias tibiales anteriores) se consideró el inferior.

El IDB se calculó en los casos en los que el ITB fue inferior a 0,9 y en aquellos en los que el ITB fue superior a 1,3 ya que nos permite obviar la falta de compresibilidad de las arterias motivada por la presencia de calcificaciones (10).
Se consideró indicador de enfermedad arterial periférica en los casos con ITB inferior a 0,9 y/o IDB inferior a 0,5.

Con todos los datos recogidos de la exploración clasificamos a los pacientes en cuatro grupos: pacientes sin neuropatía ni enfermedad arterial periférica (grupo normal), pacientes con neuropatía periférica pero sin enfermedad arterial periférica (grupo neuropático), pacientes con enfermedad arterial periférica pero sin neuropatía periférica (grupo vascular) y pacientes con ambas patologías (grupo mixto).

Se compararon las características de los pacientes del grupo normal, con las de los pacientes del grupo neuropático, con los del grupo vascular y con los del grupo mixto.

De cada uno de los pacientes además de los datos anteriormente reseñados se recogieron otra serie de variables: presencia de otros factores de riesgo cardiovascular (HTA, dislipemia y tabaquismo), presencia de complicaciones microvasculares (retinopatía y nefropatía), complicaciones macrovasculares (accidente cerebrovascular y cardiopatía isquémica), si recibían o no antiagregación y el grado de control metabólico a través del cálculo de la HbA1c media del año previo a la realización del estudio.

La hipertensión arterial fue definida como una presión arterial $\geq 140 / 90 \mathrm{mmHg}$ y/o por la toma de algún antihipertensivo. La dislipemia fue definida por la presencia de colesterol total sérico $\geq 240 \mathrm{mg} / \mathrm{dl}$ y/o triglicéridos $\geq 160 \mathrm{mg} / \mathrm{dL}$ y/o por la toma de algún hipolipemiante. Se consideró fumadores a los fumadores activos y a los exfumadores que habían abandonado el hábito tabáquico hacía menos de 10 años. La existencia de retinopatía se determinó mediante la realización de fondo de ojo y la de nefropatía mediante la determinación de microalbuminuria en orina de 8 horas. La hemoglobina glicosilada se determinó por el método de cromatografía de alta resolución (HPLC).

El análisis estadístico se realizó utilizando el programa informático SPSS versión 7.0 para Windows. Las variables cuantitativas se presentan como media \pm desviación estándar y las cualitativas como porcentaje. Las comparaciones entre las variables cuantitativas se realizaron con el análisis de las varianzas (ANOVA) ajustado con el test de Bonferroni y las de las variables cualitativas mediante el test de $\mathrm{x}^{2} \mathrm{o}$ test exacto de Fisher, según el número. Para evaluar los factores asociados de forma independiente con la presencia de neuropatía periférica, de arteriopatía periférica o de ambas patologías se utilizó un modelo de regresión logística múltiple incluyendo en el modelo aquellas variables con significación estadística en el análisis univariante $(\mathrm{p}<0,05)$.

\section{RESULTADOS}

Según los criterios de clasificación utilizados en nuestra población obtuvimos los siguientes resultados: $33,2 \%$ sin patología, $21,1 \%$ con neuropatía periférica, $21,4 \%$ con enfermedad arterial periférica y $24,3 \%$ con ambas patologías.

En la tabla I se muestran las características de los pacientes según la ausencia o presencia de factores de riesgo para el desarrollo de neuropatía y/o vasculopatía periférica. La edad media en los grupos vascular $(63 \pm 13)$ y mixto $(65 \pm 10)$ fue significativamente mayor que en el grupo normal $(55 \pm 14)$. La frecuencia de hipertensión arterial en los grupos vascular $(69,2 \%)$ y mixto $(70,3 \%)$ fue significativamente mayor que en el grupo normal $(45,5 \%)$. La frecuencia de antiagregación 
TABLA I

CARACTERÍSTICAS DE LOS PACIENTES SEGÚN LA PRESENCIA O AUSENCIA DE FACTORES DE RIESGO VASCULARES O NEUROPÁTICOS PARA EL DESARROLLO DE PIE DIABÉTICO

\begin{tabular}{lcccc}
\hline & Normal & Neuropático & Vascular & Mixto \\
\hline $\mathrm{n}$ & 101 & 64 & 65 & 74 \\
Edad (años) & $55 \pm 14,2$ & $59 \pm 11,4$ & $62,6 \pm 12,8^{* *}$ & $64,8 \pm 10,2^{* * *}$ \\
Duración (años) & $14,6 \pm 10,3$ & $17,8 \pm 11,8$ & $13,9 \pm 11,8$ & $19 \pm 12,4$ \\
HTA (\%) & 45,5 & 54,7 & $69,2^{* *}$ & $70,3^{* *}$ \\
Dislipemia (\%) & 65,3 & 51,6 & 70,8 & 51 \\
Tabaquismo (\%) & 43,2 & 32,7 & 45,9 & 41,7 \\
Antiagregación (\%) & 51,5 & 57,8 & $75,4^{* *}$ & $77^{* *}$ \\
HbA1c (\%) & 7,4 & $8,1^{*}$ & 7,6 & $8^{*}$ \\
\hline Los valores de p se refieren a la comparación del grupo normal con los
\end{tabular}
otros 3 grupos: ${ }^{*}: p<0,05 ;{ }^{* *}: p<0,01 ;{ }^{* *}: p<0,001$.

en los grupos vascular $(75,4 \%)$ y mixto $(77 \%)$ fue significativamente mayor que en el grupo normal $(51,5 \%)$.

Comparado con el grupo normal, la $\mathrm{HbA} 1 \mathrm{c}$ media fue significativamente mayor en los grupos neuropático y mixto $(7,4$ $\pm 1,2$ vs. $8,1 \pm 1,6$ y $8 \pm 1,3$ respectivamente).

La frecuencia de cardiopatía isquémica en los grupos vascular $(46,2 \%)$ y mixto $(39,2 \%)$ fue significativamente mayor $(\mathrm{p}<0,005$ y $\mathrm{p}<0,05)$ que en el grupo normal $(23,8 \%)$. La frecuencia de retinopatía en los grupos neuropático $(62,5 \%)$ y mixto $(66,2 \%)$ fue significativamente mayor $(\mathrm{p}<0,001$ y $\mathrm{p}<0,001)$ que en el grupo normal $(32,7 \%)$. La frecuencia de nefropatía en los grupos neuropático $(45,3 \%)$ y mixto $(47,3 \%)$ fue significativamente mayor $(\mathrm{p}<0,01 \mathrm{y} \mathrm{p}<0,005)$ que en el grupo normal $(24,8 \%)$. La frecuencia de accidentes cerebrovasculares no mostró diferencias significativas entre los diferentes grupos (Fig. 1).

En el análisis multivariante que evaluaba los factores que se asociaban de forma independiente con la presencia de neuropatía periférica, con enfermedad arterial periférica o con ambas patologías se observó que los factores que se asociaron de forma independiente con la presencia de neuropatía periférica fueron la hemoglobina glicada (OR: 1,38; IC95\%: 1,061,78 ) y la existencia de retinopatía (OR: 3,11; IC95\%: 1,59$6,09)$. Los factores que se asociaron de forma independiente con la presencia de enfermedad arterial periférica fueron la edad (OR: 1,04; IC95\%: 1,01-1,07) y la existencia de cardiopatía isquémica (OR: 2,52; IC95\%: 1,27-5.00). Los factores que se asociaron independientemente con la presencia concomitante de ambas patologías fueron la edad (OR: 1,09; IC95\%: 1,05-1,13), la existencia de cardiopatía isquémica (OR: 2,36; IC95\%: 1,03-5,39), la hemoglobina glicada (OR: 1,54; IC95\%: 1,14-2,07) y la existencia de retinopatía (OR: 5,68; IC95\%: 2,53-12,75).

No se encontraron diferencias significativas entre el grupo con estudio normal y los otros tres grupos con respecto al tipo de diabetes, la duración de la misma, el sexo, la presencia de tabaquismo o la existencia de dislipemia.

\section{DISCUSIÓN}

En primer lugar debemos reseñar que la alta prevalencia de pacientes con estudio patológico de nuestro trabajo no refleja la realidad de la población diabética, ya que nuestra

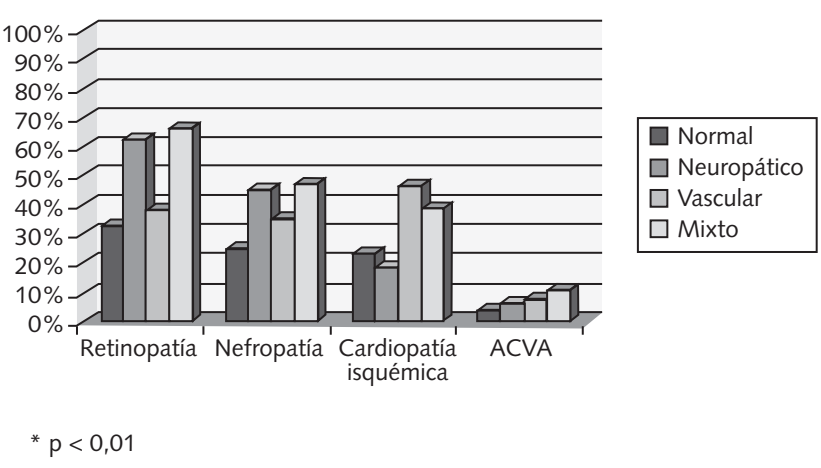

Fig. 1. Presencia de complicaciones micro y macrovasculares en la población estudiada según la presencia de factores de riesgo.

muestra pertenece a pacientes seguidos en una Unidad de Diabetes de un hospital terciario dónde se suelen concentrar los casos con más factores de riesgo y/o con peor control metabólico de la diabetes.

Según los resultados de nuestro estudio la presencia de neuropatía diabética se relaciona con un peor control glucémico y con la existencia de otras complicaciones microvasculares (retinopatía y nefropatía). Aunque se desconoce con exactitud la etiopatogenia de la neuropatía diabética existen datos epidemiológicos, experimentales y clínicos que apoyan el papel central de la hiperglucemia en la etiología de las diversas formas de neuropatía (11). Así se han propuesto como mecanismos etiopatogénicos el aumento de la glicacion no enzimática de proteínas estructurales del nervio o cambios vasculares con hipoxia endoneural debido a microangiopatía; siendo este último mecanismo el nexo etiopatogénico común con las otras complicaciones microangiopáticas de la diabetes como la retinopatía y la nefropatía .

Por otro lado, la presencia de enfermedad arterial periférica, según nuestro trabajo y como parece obvio, se relaciona con la edad, la presencia de hipertensión arterial y con la coexistencia de otras complicaciones macrovasculares como la cardiopatía isquémica.

Sin embargo, en contra de lo ya demostrado clínicamente, no hemos encontrado que factores como el tabaquismo y la dislipemia se relacionen con un incremento de la presencia de enfermedad arterial periférica.

La explicación a esta posible discrepancia con respecto a estudios previos puede residir en la forma de recogida de los datos ya que en lo que respecta al hábito tabáquico se incluyó a los exfumadores desde hace más de 10 años en el grupo de los no fumadores lo que puede haber constituido un factor de confusión sobrestimando el riesgo de los que realmente nunca han fumado (12).

En lo que respecta a la dislipemia la clasificación demasiado generalizada que hemos utilizado puede ser la responsable de no haber obtenido resultados concordantes con otros estudios (13); al no haberse tenido en cuenta de manera precisa los aspectos cuantitativos y cualitativos de la misma (hipertrigliceridemia e hipercolesterolemia).

Es sabido que los pacientes diabéticos tienen mayor riesgo cardiovascular que la población general, no en vano el infarto de miocardio es la principal causa de muerte (14). 
Este aumento de riesgo se trata de explicar por la presencia de factores de riesgo entre los que se halla el probable papel patogénico ejercido por la hiperglucemia en el desarrollo de la aterosclerosis; de forma que el riesgo cardiovascular se reduciría con un mejor control glucémico $(15,16)$.

Nuestros datos no encuentran relación entre la existencia de enfermedad arterial periférica y un peor control glucémico; hecho que podría explicarse teniendo en cuenta que el umbral de hiperglicemia necesario para evitar la aparición de complicaciones macrovasculares es inferior al referido para las complicaciones microvasculares, y sólo podríamos evitar el papel lesivo de la hiperglucemia, siendo muy estrictos en el control glucémico (17).

Como es de suponer el grupo mixto presenta los factores de riesgo de ambos grupos.
En resumen, con los datos extraídos de nuestro trabajo llegamos a la conclusión de que la aparición de neuropatía diabética se relaciona de forma directa con el control metabólico. Por otro lado concluimos que los pacientes de mayor edad, con hipertensión arterial o con manifestaciones arterioscleróticas a distintos niveles (cardiopatía isquémica) tienen mayor riesgo de presentar enfermedad arterial periférica.

Esto refuerza la idea de la necesidad de crear Unidades de pie diabético dónde se valore el riesgo vascular y neuropático que presentan los pacientes diabéticos para desarrollar el pie diabético; de esta forma podremos planificar programas de prevención y detección precoz de estas alteraciones que eviten las todavía frecuentes amputaciones de miembros inferiores.

\section{Bibliografía}

1. Sheahan MG, Hamdan AD, Veraldi JR, McArthur CS, Skillman JJ, Campbell D.R. et al. Lower extremity minor amputations: The roles of diabetes mellitus and timing of revascularization. Vasc Surg 2005; 42: 476-80.

2. The Diabetes Control and Complications Trial Research Group. The effect of intensive diabetes therapy on the development and progression of neuropathy. Ann Intern Med 1995; 122: 561-8.

3. UK Prospective Diabetes Study (UKPDS) Group. Intensive blood-glucose control with sulphonylureas or insulin compared with conventional treatment and risk of complications in patients with type 2 diabetes (UKPDS 33). Lancet 1998; 352: 837-53.

4. Tesfaye S, Stevens L, Stephenson J, Fuller JH, Plater H, Ionescu-Tirgoviste $\mathrm{C}$. et al. The prevalence of diabetic peripheral neuropathy and its relation to glycaemic control and potential risk factors: The EURODIAB IDDM complications study. Diabetologia 1996; 39: 1377-84

5. Dyck PJ, et als. Risk factors for severity of diabetic polyneuropathy. Intensive longitudinal assessment of the Rochester Diabetic Neuropathy. Study cohort. Diabetes Care 1999; 22: 1479-86

6. Epidemiology of Diabetes Interventions and Complications (EDIC): design, implementation, and preliminary results of a long-term followup of the Diabetes Control Complications Trial cohort. Diabetes Care 1999; 22: 99-111.

7. Glycemic control and cardiovascular disease-should we reassess clinical goals? (editorial). N Engl J Med 2005: 353; 25.

8. Young MJ, Boulton AMJ, Mac Leod AF, Williams DRR, Sonksen PH A multicentre study of the prevalence of diabetic peripheral neuropathy in the United Kingdom hospital clinic population. Diabetología 1993; 36: $150-4$.

9. Herranz de La Morena L. Índice tobillo brazo en la evaluación de la enfermedad arterial periférica. Av Diabetol 2005; 21: 224-6

10. Brooks B, Dean R, Patel S, Wu B, Molyneaux L, Yue DK TBI or not TBI: that is the question. It is better to measure toe pressure than ankle pressure in diabetic patients? Diabet Med 2001; 18: 528-32.

11. Reichard P, Nilsson BY, Rosenquisit U. The effect of long- term intensified insulin treatment on the development of microvascular complications of diabetes mellitus. N Engl J Med 1993; 329: 304-9.

12. Albu J, Gottlieb SH, August P, Nesto RW, Orchard TJ; Bypass Angioplasty Revascularization Investigation 2 Diabetes (BARI 2D) Trial Investigators. Modifications of coronary risk factors. Am J Cardiol 2006; 97: 41G-52G.

13. EURODIAB Prospective Complications Study Group. Vascular risk factors and diabetic neuropathy. N Engl J Med 2005; 352: 341-50.

14. Gandhi GY, Roger VL, Bailey KR, Palumbo PJ, Ransom JE, Leibson CL. Temporal trends in prevalence of diabetes mellitus in a populationbased cohort of incident myocardial infarction and impact of diabetes on survival. Mayo Clin Proc 2006; 81: 1034-40.

15. Selvin E, Marinopoulos S, Berkenblit G, et al. Meta-analysis: Glycosylated hemoglobin and cardiovascular disease in diabetes mellitus. Ann Intern Med 2004; 141: 421-41.

16. The Diabetes Control And Complications Trial/Epidemiology of Diabetes Interventions and Complications (DCCT/EDIC) Study Research Group. Intensive diabetes treatment and cardiovascular disease in patients with type 1 diabetes. N Engl J Med 2005; 353: 2643-53.

17. Lobby P, Plutzky J. Diabetic macrovascular disease. The glucose paradox? Circulation 2002; 106: 2760-3. 